\title{
MONITORING THE NUTRITIONAL STATUS OF PATIENTS WITH PRESSURE ULCERS USING THE CONTROLLING NUTRITIONAL STATUS METHOD
}

\author{
H. Kishimoto
}

\begin{abstract}
Objectives: To evaluate methods for monitoring the nutritional status of patients with pressure ulcers (PU). Design: A retrospective case series study. Setting and Participants: Twenty-nine patients (mean age, 80.6 years; 10 men) with PU admitted to the acute care ward of Namegata District General Hospital, Japan, were recruited. Measurements: The nutritional status of the patients was evaluated using the controlling nutritional status (CONUT) method. The wound assessment tool DESIGN-R was used to evaluate the wound state of PU. The rate of improvement in CONUT score (CR) was calculated for each patient from the CONUT values at the beginning and end of the observation period divided by the number of days. In addition, the rates of improvement of the serum albumin level (AR) and DESIGN-R level (DR) were also calculated. Results: There was a moderately strong correlation between $\mathrm{CR}$ and DR that was significant $(\mathrm{r}=0.60, \mathrm{p}<0.01)$; however, the correlation between AR and DR was weaker and not significant $(\mathrm{r}=0.36, \mathrm{p}=0.056)$. Conclusion: Improvement in nutritional status evaluated by CONUT correlated with the improvement in ulcer severity evaluated by DESIGN-R in patients with PU. The CONUT method is suitable for nutritional status monitoring of the patient with PU in a hospital setting.
\end{abstract}

Key words: Pressure ulcer, controlling nutritional status (CONUT), DESIGN-R, monitoring.

\section{Introduction}

The prevention and amelioration of malnutrition are essential in the management of patients with or at risk of a pressure ulcer (PU). There is a requirement for an index for clinically monitoring the nutritional state of patients with PU; however, there is no definite recommendation about which tool to use for this. The Japanese Society of Pressure Ulcers (JSPU) indicated in their guidelines for the prevention and management of PU (4th edition) (1) that serum albumin (Alb) level, rate of decline in weight, nutritional intake, Mini Nutritional Assessment ${ }^{\circledR}($ MNA $®)$ score $(2,3)$, controlling nutritional status (CONUT) (4), and subjective global assessment (SGA) score (5) may be used as indexes to screen for undernutrition that could become a risk factor for PU. The indexes are the standard methods for screening the nutritional status of patients with or at a risk of PU, but there is no standard method to monitor the nutritional status of a patient during PU treatment.

Department of Rehabilitation Medicine, Ibaraki Prefectural University of Health Sciences Hospital

Corresponding Author: Hiroshi Kishimoto, 4733 Ami Ami-town Inashikigun, Ibaraki 300-0331, Japan, Phone: +81 298889200, Fax: +81 298402981, E-mail: hiroshi.k.dmd@gmail.com
In contrast, the wound assessment tool DESIGN-R involves a ratio scale and can be used for comparing the severity of PU in different cases (6-8). This scale's predictive validity has been assessed, and it has become the gold standard rating system for PU in Japan (1, 6-8).

Out of the nutritional evaluation indexes recommended in the JSPU guidelines, in this study, we selected serum Alb level and CONUT score for convenience and objectivity of their measurement. Correlations between improvements in these indexes and healing of PU were investigated to assess the suitability of these indexes for monitoring the nutritional status of patients with PU.

\section{Materials and methods}

\section{Patients}

In this retrospective study, we reviewed 96 patients with PU admitted to the acute care ward of Namegata District General Hospital, Japan, (mean hospital stay duration, 14 days) between May 2013 and June 2014. Nine patients who were observed for $<14$ days and 58 patients for whom CONUT score was not calculated at the beginning and end of the observation period were 
excluded from the study. Data for the remaining 29 patients were analyzed.

\section{DESIGN-R}

The wound assessment tool DESIGN-R, recommended by JSPU (1, 6-8), is used in our institution for the evaluation of the wound state of PU. The task team for PU, which includes a dermatologist and nurses specialized in dermatology, evaluated the state of PU once a week. The DESIGN-R scores at the beginning and end of the observation period were obtained from each patient's medical records.

\section{Evaluation of Nutritional Status}

In this study, a modified form of CONUT was used to evaluate the nutritional status of the patients. This form incorporates the corrections by Hashimoto et al. (9) and takes into account the serum Alb level being measured in our institution using the modified bromocresol purple method rather than by the bromocresol green method used in the original work. The CONUT scores and serum Alb levels at the beginning and end of the observation period were obtained from each patient's medical records.

\section{Evaluation of the improvement in the nutritional status and wound state}

The rate of improvement in the CONUT score (CR) was calculated for each patient as the difference between the scores at the beginning and end of the observation period divided by the length of the observation period in days. Rates of improvement in serum Alb level (AR) and DESIGN-R score (DR) were calculated in the same way.

\section{Statistical analysis}

Statistical analyses were performed using the SPSS software (version 22.0, IBM). Spearman's rank correlation coefficients were calculated for analyzing the correlation between $C R$ and DR and between AR and DR. A p-value $<0.05$ was considered statistically significant.

\section{Results}

Demographic and clinical characteristics of the 29 patients (mean age, 80.6 years; 10 males) are shown in Table 1. Eleven patients were in a chronic condition; eight, acute; eight, postoperative; and two, palliative. The mean observation period was 32.8 days. The table also shows the mean CONUT, Alb, and DESIGN-R levels for the beginning and end of the observation period; lower scores for CONUT and DESIGN-R indicate improvement in status. Scatter diagrams of CR vs. DR and AR vs. DR are plotted in Figure 1a and $b$. There was a moderately strong correlation between CR and DR $(r=0.60, p<0.01)$. The correlation between AR and DR was weaker and not significant $(\mathrm{r}=0.36, \mathrm{p}=0.056)$.

Table 1

Patients' demographic and clinical characteristics

\begin{tabular}{|c|c|c|}
\hline & Categories & Values \\
\hline Age, mean (SD) & & $80.6( \pm 9.0)$ \\
\hline \multirow[t]{2}{*}{ Sex, n } & Male & 10 \\
\hline & Female & 19 \\
\hline \multirow[t]{4}{*}{ Condition, $\mathrm{n}$} & Acute & 13 \\
\hline & Chronic & 6 \\
\hline & Postoperative & 7 \\
\hline & Palliative & 3 \\
\hline \multirow[t]{7}{*}{ Comorbidity, $\mathrm{n}$} & Musculoskeletal & 10 \\
\hline & Infectious & 7 \\
\hline & Nervous & 3 \\
\hline & Neoplasms & 2 \\
\hline & Mental/behavioral & 3 \\
\hline & Circulatory & 2 \\
\hline & Respiratory & 2 \\
\hline $\begin{array}{l}\text { Observation period, mean (SD, } \\
\text { days) }\end{array}$ & & $32.8( \pm 19.7)$ \\
\hline \multirow{2}{*}{$\begin{array}{l}\text { Serum albumin level }(\mathrm{g} / \mathrm{dl}) \text {, mean } \\
\text { (SD) }\end{array}$} & Beginning & $2.20( \pm 0.56)$ \\
\hline & End & $2.44( \pm 0.57)$ \\
\hline \multirow[t]{2}{*}{ CONUT score, mean (SD) } & Beginning & $8.66( \pm 2.44)$ \\
\hline & End & $6.79( \pm 2.92)$ \\
\hline \multirow[t]{2}{*}{ DESIGN-R score, mean (SD) } & Beginning & $19.2( \pm 11.5)$ \\
\hline & End & $13.9( \pm 11.7)$ \\
\hline
\end{tabular}

Figure 1

Scatter diagram of the rate of improvement of ulcer severity as measured by DESIGN-R (DR) and the rate

of improvement of nutritional status as measured by CONUT (CR) in 29 patients with pressure ulcers. Negative values indicate improvement. The Spearman's coefficient of correlation is $0.60(p<0.01)$

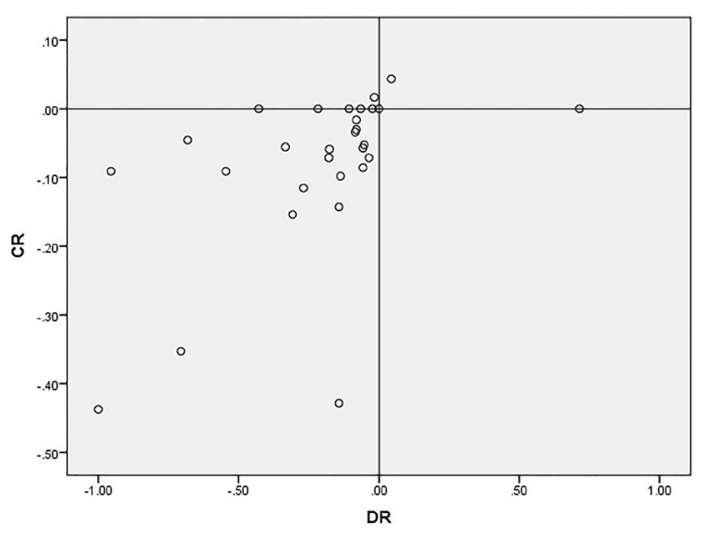




\section{Figure 2}

Scatter diagram of the rate of improvement of ulcer severity as measured by DESIGN-R (DR) and the rate of improvement of nutritional status as measured by serum albumin levels (AR) in 29 patients with pressure ulcers. Negative values of DR indicate improvement. The Spearman's coefficient of correlation is $0.36(p=0.056)$

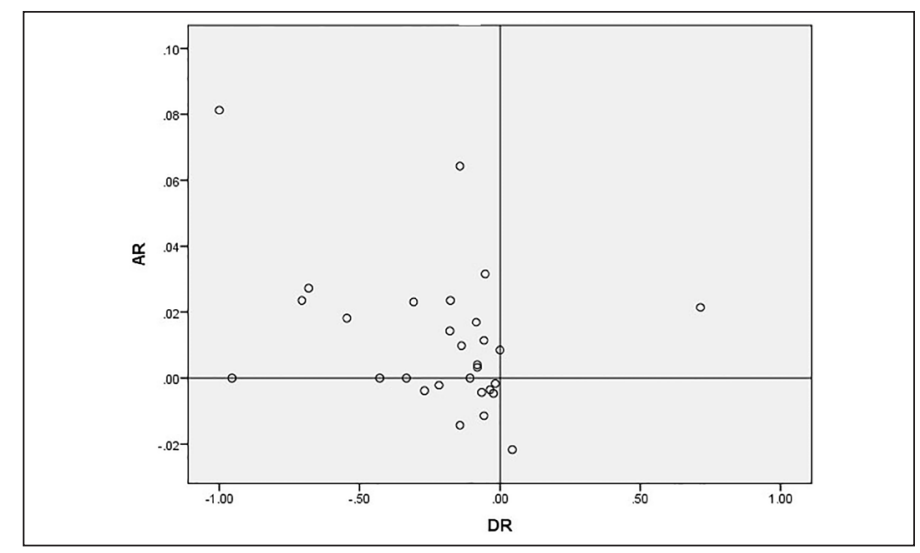

\section{Discussion}

In this study, we focused on the changes over the observation period in CONUT, Alb, and DESIGN-R, and found a statistically significant correlation between the rates of improvement of the CONUT and DESIGN-R scores. Therefore, when the state of PU is evaluated by DESIGN-R, the CONUT method is one of the useful nutritional status rating systems in the hospital setting. Iizaka et al. reported that the serum Alb level is not associated with the healing of PUs, except for superficial PUs in the acute condition (11). Also in this study, we did not find a significant correlation between AR and DR, although a weak correlation close to statistical significance was observed. It is speculated that CONUT method is better than Alb as a nutritional status index for patients with PUs because not only the competency of protein synthesis but also the competency of lipid synthesis and immunocompetence are evaluated by cholesterol and lymphocytes in the CONUT method.

Apart from the retrospective nature of this study, there were two other limitations. First, the number of patients was small. Second, a potential bias may have resulted from including only the cases for which CONUT was calculated at the beginning and end of the observation period. A prospective study using CONUT and DESIGN-R should be investigated in the future.
In conclusion, a correlation was found between the change in nutritional status as assessed using CONUT and the change in ulcer severity as assessed using DESIGN-R in patients with PU. The CONUT method is suitable for monitoring the nutritional status of patients with PU in a hospital setting.

Acknowledgment: I would like to express my sincere gratitude to the Pressure Ulcer Task Team and Nutrition Support Team of Namegata District General Hospital, Japan, for their help. I would also like to thank the following individuals: Kazuyuki Ueno (clinical laboratory technologist); Sanae Numata, Mariko Nakata, Yayoi Sugaya (nurses); Yutaka Ando (pharmacologist); Sayaka Nakajima, Yuko Tayama (registered dietitians); and Kyoko Iida (speech therapist).

Conflict of interest: I have no conflict of interest to declare.

Ethics statement: This study was conducted in accordance with the Declaration of Helsinki and was approved by the Ethics Committee of Namegata District General Hospital, Japan. Because this was a retrospective study, written informed consent was not obtained from the patients.

\section{References}

1. The Japanese Society of Pressure Ulcers Guideline Revision Committee. JSPU guidelines for the prevention and management of pressure ulcers (4th Ed.) Jpn J PU (in Japanese) 2015;17:487-557

2. Vellas B, Guigoz Y, Garry PJ, Nourhashemi F, Bennaham D, Lauque S, Albarede JL. The Mini Nutritional Assessment (MNA) and its use in grading the nutritional state of elderly patients. Nutrition 1999;15:116-122

3. Rubenstein LZ, Harker JO, Salvà A, Guigoz Y, Vellas B. Screening for undernutrition in geriatric practice: Developing the short-form Mini-Nutritional Assessment (MNA-SF). J Gerontol A Biol Sci Med Sci 2001;56:M366-72

4. Ignacio de Ulíbarri J, González-Madroño A, de Villar NG. CONUT: a tool for controlling nutritional status. First validation in a hospital population. Nutr Hosp 2005; 20:38-45

5. Detsky AS, McLaughlin JR, Baker JP, Johnston N, Whittaker S, Mendelson RA, Jeejeebhoy KN. What is subjective global assessment? JPEN 1987;11:8-13

6. Matsui Y, Furue M, Sanada H, Tachibana T, Nakayama T, Sugama J, Furuta $\mathrm{K}$ et al. Development of the DESIGN-R with an observational study: An absolute evaluation tool for monitoring pressure ulcer wound healing. Wound Repair Regen 2011;19:309-315

7. Iizaka S, Sanada H, Matsui Y, Furue M, Tachibana T, Nakayama T, Sugama $\mathrm{J}$ et al. Predictive validity of weekly monitoring of wound status using DESIGN-R score change for pressure ulcer healing: a multicenter prospective cohort study. Wound Repair Regen 2012;20:473-81

8. Sanada H, Iizaka S, Matsui Y Furue M, Tachibana T, Nakayama T, Sugama $\mathrm{J}$ et al. Clinical wound assessment using DESIGN-R total score can predict pressure ulcer healing: Pooled analysis from two multicenter cohort studies. Wound Repair Regen 2011;19:559-567

9. Hashimoto N, Katayama K, Imura T Kurose T, Kitayama F, Hayase M, Tatsuhira $\mathrm{H}$ et al. The practical problem of the clinical nutrition indices estimated from the serum albumin (Alb) values.:their partial but significant discrepancy between the conventional and improved Alb measurement methods. Joumyakukeichoueiyou (The journal of Japanese society for parenteral and enteral nutrition, in Japanese) 2013;28:1091-1099

10. National Pressure Ulcer Advisory Panel, European Pressure Ulcer Advisory Panel and Pan Pacific Pressure Injury Alliance. Prevention and Treatment of Pressure Ulcers: Quick Reference Guide. Emily Haesler (Ed.). Cambridge Media: Osborne Park, Australia; 2014

11. Iizaka S, Sanada H, Matsui Y Furue M, Tachibana T, Nakayama T, Sugama $\mathrm{J}$ et al. Serum albumin level is a limited nutritional marker for predicting wound healing in patients with pressure ulcer: Two multicenter prospective cohort studies. Clin Nutr 2011;6,738-745 